\title{
Anomaly detection on in-home activities data based on time interval
}

\author{
Soon-Chang Poh, Yi-Fei Tan, Soon-Nyean Cheong, Chee-Pun Ooi, Wooi-Haw Tan \\ Faculty of Engineering, Multimedia University, Malaysia
}

\begin{tabular}{l}
\hline Article Info \\
\hline Article history: \\
Received Sep 27, 2018 \\
Revised Jan 21, 2019 \\
Accepted Feb 5, 2019 \\
\hline Keywords: \\
Anomaly detection \\
Behavioral changes \\
Changes in routine \\
Elderly care \\
In-home activities
\end{tabular}

\begin{abstract}
The world population of the elderly is expected to have a continuous growth and the number of elderly living in solitude is also expected to increase in the coming years. As our health decline with age, early detection of possible deterioration in health becomes important. Behavioral changes in in-home activities can be used as an indicator of health decline. For example, changes in routine of in-home activities. Past research mainly focused on detecting anomalies in routine of each type of in-home activities individually. In this paper, an anomaly detection model to detect changes in routine of in-home activities collectively for a day is proposed. The experiment was evaluated with an existing public dataset. The experimental results demonstrated that the anomaly detection model performed well on unseen testing data with an accuracy of $94.44 \%$.
\end{abstract}

Copyright $(2019$ Institute of Advanced Engineering and Science. All rights reserved.

\section{Corresponding Author:}

Soon-Chang Poh, Faculty of Engineering, Multimedia University, Persiaran Multimedia, 63100, Cyberjaya, Selangor, Malaysia.

Email: psoonchang@gmail.com

\section{INTRODUCTION}

The United Nations predicted the world population of the elderly (65 years old and above) is expected to accelerate in the coming decades [1]. In Malaysia, one out of five of the whole population was predicted to be elderly (60 years old and above) by 2040 [2]. There is an increasing number of elderly end up living in solitude [2]. Hence, it is conjectured that there will be a significant population of solitude elderly in the future. Solitude elderly without care have a higher risk of mortality due to lack of care and monitoring of their health conditions [3-5]. One of the solutions is to hire caregivers for consistent monitoring and care, but the cost is often quite expensive for long-term care. An alternative option is to use the Internet of Things (IoT) technology in monitoring the elderly's daily activities or condition.

There are several research focusing on in-home activity recognition and the two main types of activity recognition are sensor-based and vision-based activity recognition. The sensor-based activity recognition uses sensors such as accelerometer installed on a wearable device such as smart watch [6-9]. The vision-based activity recognition uses camera to capture video of human subject's activity as input data [10]. Most of the researchers focused on sensor-based activity recognition used machine learning algorithm for classification. Some recent papers [11-14] used deep learning algorithm for classification. In addition to the two main types of activity recognition, a new activity recognition paradigm based on Internet of Things (IoT) using wireless consumer products was also proposed [15]. Regardless of the methods, the collected data can be categorized into several in-home activities using the activity recognition software in the format of "Date", "Time" and "Type of activity". By observing one's in-home activities records, his or her usual routine of in-home activities may be modeled and the anomalies can be detected.

There are two main types of anomalies that can be detected from in-home activities data namely sequence anomaly and time anomaly. Sequence anomaly refers to abnormalities in sequence pattern of in- 
home activities data. Some researchers used machine learning algorithm such as Hidden Markov Model (HMM) [16] and Long Short-Term Memory (LSTM) [17] for sequence anomaly detection. In [18], Forkan and colleagues introduced a sequence anomaly detection method based on HMM with averagely $90 \%$ accuracy on an artificial dataset. In [19], Tan and her peers proposed LSTM neural network for in-home activities sequence anomaly detection with a comparison with HMM. Due to the nature of the problem, the data size of in-home activities is limited in range of hundreds, which is not big relative to datasets of other data analysis task. This limitation in data size results in complex machine learning algorithm which leverages on big data does not perform well on this task. In [20], Poh and colleagues introduced a simple alternative method using a database to detect sequence anomaly in in-home activities with a test accuracy of $90.79 \%$.

Time anomaly refers to change in usual routine of an in-home activity. Past works focused on detecting anomalies for each type of in-home activities individually and the methods includes statistical method [18] and DBSCAN clustering-based anomaly detection [21]. In [18], Forkan and colleagues also demonstrated a statistical method based on normal distribution for time anomaly detection. They assumed each type of in-home activity at starting time was normally distributed and the distribution is split into several regions of different degree of abnormality. Their technique was performed on an artificial data and it showed an accuracy above $90 \%$ generally. In [22], Hoque and colleagues focused on reducing false alarms in clustering-based anomaly detection on in-home activities with rule-based approach. In this work, the authors proposed to cluster each type of activity based on time features such as starting time and duration with a clustering algorithm known as DBSCAN. For anomaly detection, an activity is classified as abnormal if it is not within 2 standard deviations from centers of all the clusters. They successfully reduced false positives and false negatives by at least $46 \%$ and $27 \%$ respectively.

In addition to the two main types of anomalies, several researchers used in-home activities to detect specific abnormal behaviour of dementia patients and mild cognitive impairment patients [23-24]. In [23] and [24], the anomalies to be detected were the sequences of actions which were unique to the dementia patients and these sequences of actions were defined by medical experts.

In this paper, an anomaly detection model based on time interval categorizing is proposed to detect changes in routine of in-home activities collectively for a day. The rest of this paper is organized as follows. In Section 2, details for methodology are discussed. Section 3 shows the experimental results and analysis and Section 4 gives the conclusions and future work.

\section{METHODOLOGY}

Figure 1 illustrates the procedure designed to build the anomaly detection model using historical data and artificial data. The following sections discuss each steps of the procedure including data collection, data preparation, modeling and model evaluation.

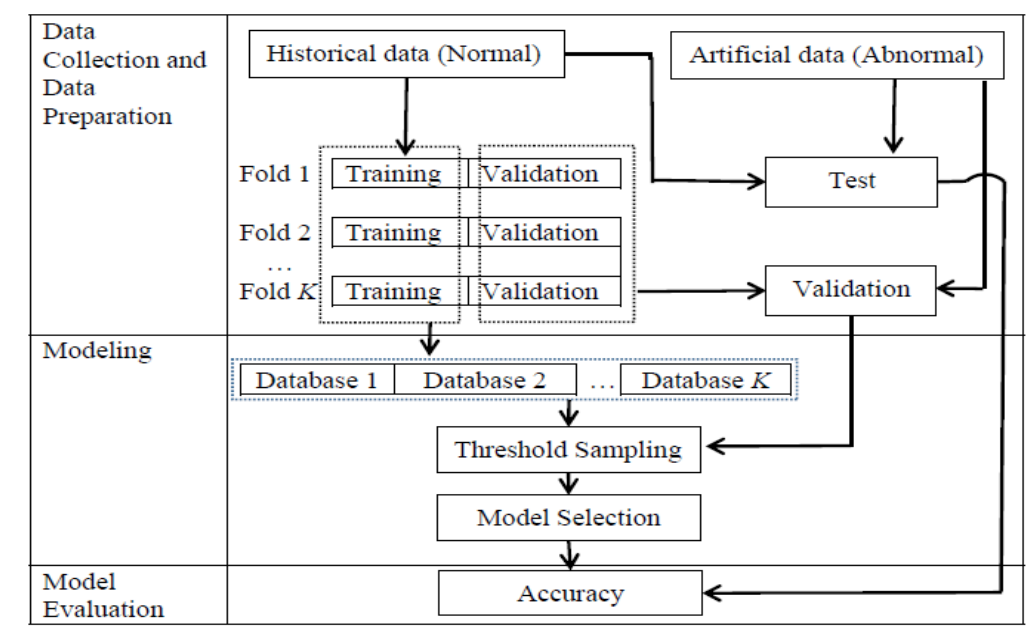

Figure 1. Framework of analysis

\subsection{Data Collection}

The public dataset used are from CASAS, Washington State University [25]. It contains 220 days of sensors data of a volunteer adult from November 2010 to June 2011. Figure 2 shows an excerpt of the 
dataset. It consists of several attributes such as date, time, sensor events, types of activity and state of activity. An example of sensor event is "M003 ON" which means a motion sensor with id, M003 is on. Besides that, the state of activity comes with two labels which are "begin" and "end". "begin" means beginning of an activity and "end" means ending of an activity. In Figure 2, it is shown that some parts of the dataset are annotated with activities such as "Sleeping" and "Bed_to_Toilet. In total, there are 11 types of activities including "Meal preparation", "Relax", "Eating", "Work", "Sleeping", "Wash dishes", "Bed_to_Toilet", "Enter home", "Leave home", "Housekeeping" and "Respirate".

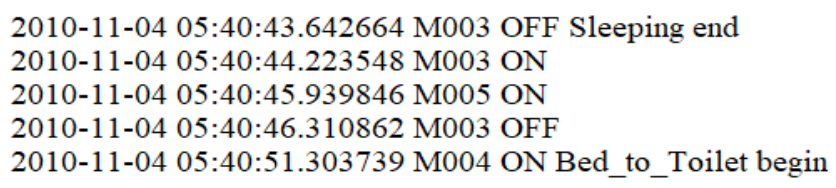

Figure 2. Excerpt of CASAS dataset

In this research, collection of all the activities happened in a day is considered as a single data instance. For anomaly detection, the data instances need to be categorized into two classes namely "normal" and "abnormal". The public dataset is considered as historical data (normal data). On the other hand, abnormal data is artificially generated. Each abnormal data instance is generated by circular shifting each inhome activities of a normal data instance by 4 hours to simulate changes in routine of in-home activities collectively for a day.

\subsection{Data Preparation}

Data preparation has 3 steps which are data processing, noise removal and data partitioning. Data processing is a step which processes the data into desired format such that it can fulfill the model requirement. Noise refers to data which has characteristics uncommon to the rest of the dataset. Some portion of the data collected contains unwanted noise. If this type of data is included in building a model, then they will affect the performance of the model. Thus, this type of data needs to be removed. During data partitioning, the processed and cleaned data are partitioned into training, validation and test set for modeling.

\subsubsection{Data Processing}

The type of data needed to build the anomaly detection model is historical records of in-home activities. Therefore, some part of the dataset which are without activity annotation were removed. The remaining data consists of only four attributes including date, time, types of activity and state of activity as shown in Figure 3.

$\begin{array}{llll}\text { 2010-11-04 } & 00: 03: 50.209589 & \text { Sleeping } & \text { begin } \\ \text { 2010-11-04 } & 05: 40: 43.642664 & \text { Sleeping } & \text { end } \\ \text { 2010-11-04 } & 05: 40: 51.303739 & \text { Bed_to_Toilet } & \text { begin } \\ \text { 2010-11-04 } & 05: 43: 30.279021 & \text { Bed_to_Toilet } & \text { end }\end{array}$

Figure 3. Excerpt of data in desired format

\subsubsection{Noise Removal}

There are 3 types of noise in this dataset. For a small portion of the dataset, the "begin" and "end" of an activity are within "begin" and "end" of another activity or overlaps with "begin" and "end" of another activity. In addition, the length of the data instances is varying, and some data instances have lengths shorter by an order of 10 compared to the rest. Lastly, the activity "Respirate" only appeared six times in the dataset. These types of noises were removed resulting in only a 176 days dataset or 176 data instances remained.

\subsubsection{Data Partitioning}

The 176 data instances are normal data which were divided into training, validation and test set. Firstly, $10 \%$ of the normal data instances were partitioned into test set. Then, the rest of the normal data instances were partitioned using $\mathrm{K}$ fold cross validation. In $\mathrm{K}$ fold cross validation, the data instances are randomly partitioned into $\mathrm{K}$ partitions and each partition should contain an equal number of data instances. 
Each of the partition can be used as validation data once and the remaining $\mathrm{K}-1$ data instances can be used as training data. This results in $\mathrm{K}$ random folds or combinations of normal training and validation data.

In the experiment, $\mathrm{K}=8$ was used and the details of normal data partitioning is listed in Table 1. Firstly, 18 data instances were randomly chosen and partitioned into test set for each of the fold. The size of remaining data is 158 and not evenly divisible by $\mathrm{K}=8$. As a result, the ratio of training to validation size is different for some of the folds. For example, the ratio for fold 1 to 5 is 138:20 and that of the rest is 139:19. However, the difference in ratio does not affect the results as the sizes are only different by one. The sizes of abnormal validation and testing data instances are equal to those of normal validation and testing data instances.

Table 1. Details of Normal Data Partitioning

\begin{tabular}{|c|c|c|c|}
\hline old & Training size & Validation size & Test size \\
\hline & 138 & 20 & 18 \\
\hline & 138 & 20 & \\
\hline & 138 & 20 & \\
\hline & 138 & 20 & \\
\hline & 138 & 20 & \\
\hline & 139 & 19 & \\
\hline & 139 & 19 & \\
\hline & 139 & 19 & \\
\hline
\end{tabular}

\subsection{Modeling}

As shown in Figure 4, the proposed anomaly detection model consists of two components including database and anomaly detector. Each anomaly detection model has 2 parameters which are the fold of training set used to train its database and the threshold $\tau$ of the anomaly detector. In this research, 80 models were built using different folds of training set and varying threshold choices. The performance and reliability of an anomaly detection model varies with its parameters. The purpose of modeling is to get the best model out of all the 80 models. Subsection 2.3.1 includes the description of the anomaly detection model and its training process. Subsection 2.3 .2 gives the method to systematically sample the threshold choices to build different models. Subsection 2.3.3 discusses the method to choose the best model.

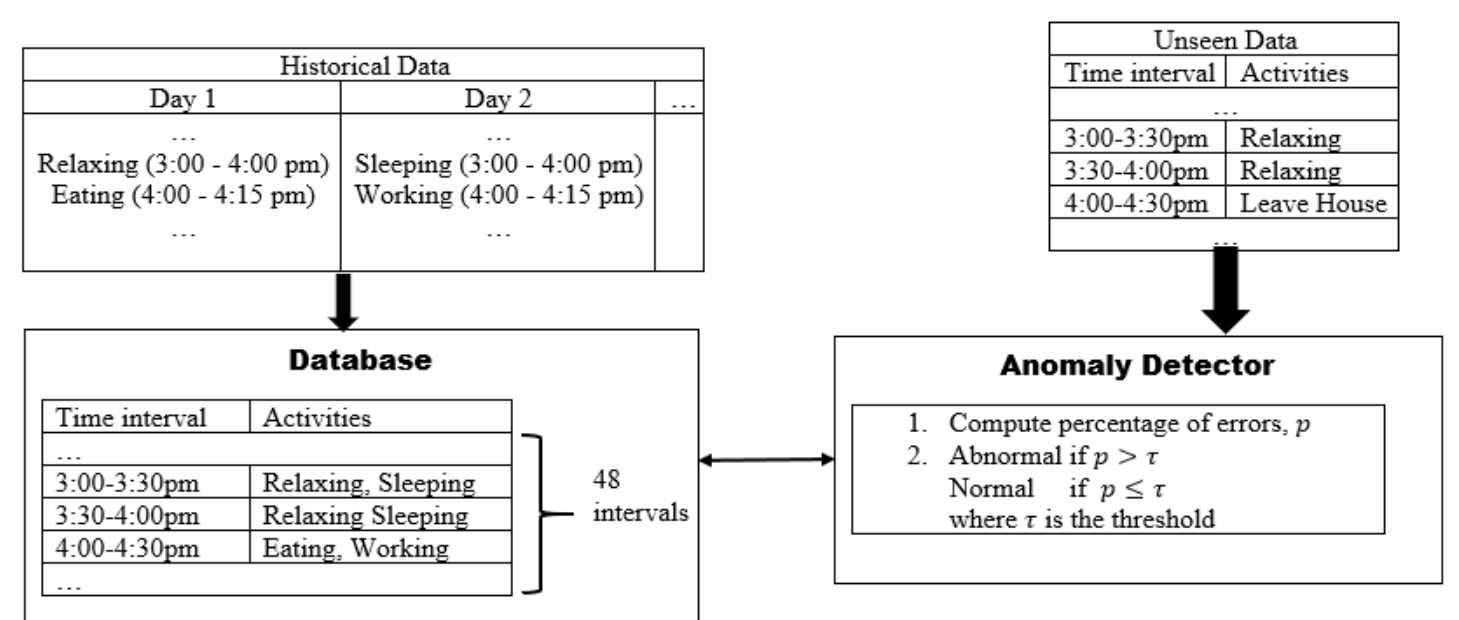

Figure 4. Overview of the anomaly detection model

\subsubsection{Training Process}

The first component of the proposed model is the database. The database models a user's normal daily routine based on time interval in a day. The database has 48 time interval categories, each for a unique 30-minutes interval in a day (e.g., 9:00-9:30 am). During the training, each of the activities in the training data instances is categorized based on the time intervals in a day and then saved into the database. For an activity that spans across several time intervals, it is saved into every time interval category it spans across. 
For example, in Figure 5, the relaxing activity spans across two 30-minutes time intervals, this activity will be saved into both the time interval categories, 3:00-3:30 pm and 3:30-4:00 pm. In this research, we used $\mathrm{K}=8$ and trained 8 different databases with different folds of training set.

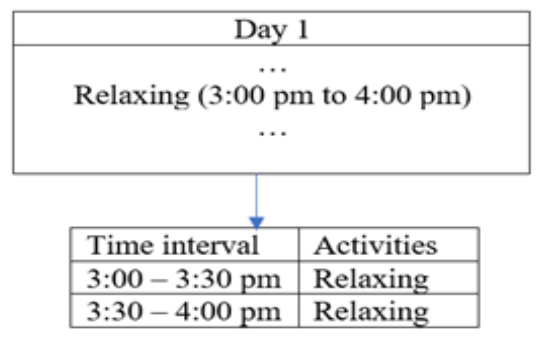

Figure 5. An activity that spans across two time interval categories

The second component of the model is the anomaly detector. Anomaly detector decides whether an unseen data instance is normal or abnormal. The first step of anomaly detection for is to check for errors in the unseen data instance by comparing it with the database. An error is defined as an activity that happens during a time interval in the data instance but it is not recorded in the respective category in the database. Then, the percentage of errors, $p$ for the unseen data instance is computed using following equation:

$$
\mathrm{p}=\frac{\text { Number of Errors }}{\text { Total number of activities }} \times 100 \%
$$

The data instance is categorized as abnormal if its percentage of errors $\mathrm{p}$ is more than the threshold $\tau$. On the other hand, it is categorized as normal if its $p$ is less than or equal to $\tau$. Threshold $\tau$ is one of the parameters of the model and the ways to sample threshold is given in the next subsection.

\subsubsection{Threshold Sampling}

The threshold $\tau$ of the model is a percentage of errors, $p$ which separates percentages of errors of normal data instances and abnormal data instances. A percentage of errors, $p$ is computed for each data instance in the validation set. The minimum and maximum of the calculated percentages of errors of every data instances in the validation set are taken as minimum and maximum of the range of threshold $\tau$. Then, T values $\left(\tau=\left\{\tau_{1}, \tau_{2}, \ldots \ldots, \tau_{\mathrm{T}}\right\}\right)$ are linearly sampled from that range as threshold choices.

\subsubsection{Model Selection}

We used $\mathrm{T}=10$ for threshold selection and trained $\mathrm{K}=8$ databases. A total of 80 different models using different database and threshold $\tau$ were created. To choose the best model, an evaluation metric, F1 score is used. The F1 score of each model is derived from Confusion Matrix which was adopted in this research to study model performance. With assistance of the definition of Confusion Matrix in Table 2, 2 evaluation metrics including precision and recall can be derived as below:

Table 2. Confusion Matrix

\begin{tabular}{|c|c|c|c|c|}
\hline & & \multicolumn{3}{|c|}{ Predicted } \\
\hline & & Positive & & Negative \\
\hline \multirow[t]{4}{*}{ Actual } & Positive & True positive, TP: & & False negative, FN: \\
\hline & & $\begin{array}{l}\text { Abnormal data instances correctly classified } \\
\text { abnormal }\end{array}$ & as & Abnormal data instances misclassified as normal \\
\hline & Negative & False positive, FP: & & True negative, TN: \\
\hline & & Normal data instances misclassified as abnormal & & Normal data instances correctly classified as normal \\
\hline
\end{tabular}

$$
\begin{aligned}
& \text { precision }=\frac{\mathrm{TP}}{\mathrm{TP}+\mathrm{FP}} \\
& \text { recall }=\frac{\mathrm{TP}}{\mathrm{TP}+\mathrm{FN}}
\end{aligned}
$$


where precision is a ratio of true positive to predicted positive and recall is a ratio of true positive to actual positive of a model. F1 score is a balanced combination of precision and recall and it can be calculated using the following equation:

$$
\mathrm{F} 1=\frac{2 \cdot \text { precision } \cdot \text { recall }}{\text { precision }+ \text { recall }}
$$

We calculated F1 score of all the 80 models (different fold and threshold $\tau$ ) and select the model with the highest F1 score as the best model.

\subsection{Model Evaluation}

Model evaluation is the step of evaluating performance of the best model on an unseen test set using the evaluation metric, accuracy. It is computed using following equation:

$$
\text { accuracy }=\frac{\mathrm{TP}+\mathrm{TN}}{\mathrm{TP}+\mathrm{FP}+\mathrm{TN}+\mathrm{FN}}
$$

\section{RESULTS AND ANALYSIS}

For each of the 80 models, a percentage of errors, $\mathrm{p}$ was calculated for each of the data instance in the validation set. Then, the minimum and maximum of all the calculated percentages of errors are $0 \%$ and $14.89 \%$. 10 values were linearly sampled from the range $[0 \%, 14.89 \%]$ and they are $0 \%, 1.65 \%, 3.31 \%$, $4.96 \%, 6.62 \%, 8.27 \%, 9.93 \%, 11.58 \%, 13.24 \%$ and $14.89 \%$.

Figure 6 shows the F1 score plot for each of the 80 models with different fold and $\tau$. From eye estimation, the models with threshold choices between $3 \%$ and $6 \%$ has the among the highest F1 scores. The best model has parameters fold $=7$ and $\tau=3.31 \%$ and the highest F1 score, $91.89 \%$.

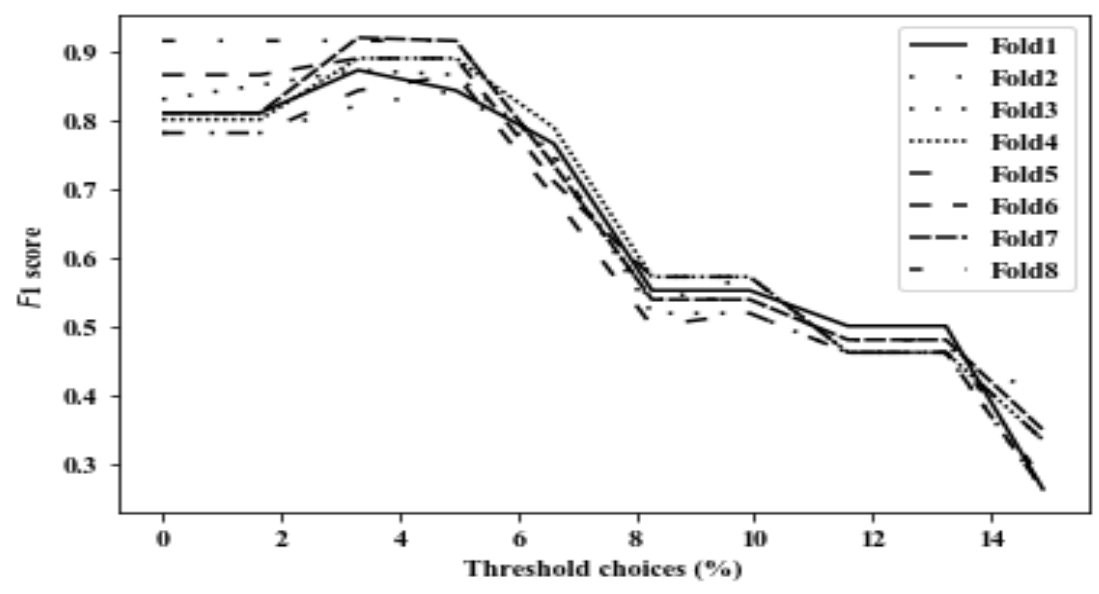

Figure 6. F1 score vs. threshold choices for $\mathrm{K}=8$

From Table 3, it is shows that the precision, recall, F1 score and accuracy of the best model evaluated on validation and unseen testing data are at least $88.89 \%$. In addition, accuracy of the model on validation and testing data are at least $92.11 \%$ and has a small difference of $2.33 \%$. This demonstrates that the model performs excellently with a high accuracy and generalizes well to unseen data. Generalizing well to unseen data is important as it guarantees consistent performance of the model when it is deployed.

Table 3. Evaluation Metrics for the Best Model

\begin{tabular}{cccc}
\hline Evaluation metrics & Validation & Testing & Difference \\
\hline Precision & $94.44 \%$ & $100 \%$ & $5.56 \%$ \\
Recall & $89.47 \%$ & $88.89 \%$ & $0.58 \%$ \\
F1 score & $91.89 \%$ & $94.12 \%$ & $2.23 \%$ \\
Accuracy & $92.11 \%$ & $94.44 \%$ & $2.33 \%$ \\
\hline
\end{tabular}




\section{CONCLUSIONS AND FUTURE WORK}

For monitoring behavioral changes of the elderly living alone at home, an anomaly detection model which can detect changes in routine of in-home activities was proposed. The experiment conducted with CASAS public dataset reveals excellent performance in terms of accuracy, precision and recall. This demonstrated that the anomaly detection model is effective in finding anomalies due to changes in routine of in-home activities collectively in a day. Currently the proposed method is mainly trained based on the activity in certain intervals of time from day to day. The correlation between the activities in the consecutive time interval may be investigated and studied to improve the model.

\section{ACKNOWLEDGEMENTS}

This work was funded by the TM Research \& Development (TM R\&D) Grant. The dataset was obtained from public dataset from CASAS, Washington State University.

\section{REFERENCES}

[1] United Nations (2015). World Population Ageing. [online] Available at: http://www.un.org/en/development/desa/population/publications/pdf/ageing/WPA2015_Report.pdf

[2] The Star (2017). Growing demand for retirement villages. [online] Available at: https://www.thestar.com.my/news/nation/2017/07/09/growing-demand-for-retirement-villages-the-demand-forretirement-villages-in-malaysia-can-only-go-up/.

[3] T. P. Ng, A. Jin, L. Feng, M. S. Z. Nyunt, K. Y. Chow, L. Feng and N.P. Fong, "Mortality of older persons living alone: Singapore Longitudinal Ageing Studies," BMC Geriatrics, vol. 15, no. 1, pp. 126-126, 2015.

[4] O. D. Lara and M. A. Labrador, "A Survey on Human Activity Recognition using Wearable Sensors," IEEE Communications Surveys and Tutorials, vol. 15, no. 3, pp. 1192-1209, 2013.

[5] Bux, P. Angelov and Z. Habib, "Vision Based Human Activity Recognition: A Review", Advances in Computational Intelligence Systems. Advances in Intelligent Systems and Computing, vol. 513, pp. 341-371, 2016.

[6] L. Bao and S. S. Intille, "Activity Recognition from User-Annotated Acceleration Data," International Conference on Pervasive Computing, pp. 1-17, 2004.

[7] G. M. Weiss, J. L. Timko, C. M. Gallagher, K. Yoneda and A. J. Schreiber, "Smartwatch-based activity recognition: A machine learning approach," 2016 IEEE-EMBS International Conference on Biomedical and Health Informatics (BHI), Las Vegas, NV, 2016, pp. 426-429.

[8] H. Huang, X. Li and Y. Sun, "A triboelectric motion sensor in wearable body sensor network for human activity recognition," 2016 38th Annual International Conference of the IEEE Engineering in Medicine and Biology Society $(E M B C)$, Orlando, FL, 2016, pp. 4889-4892.

[9] D. Tian, X. Xu, Y. Tao and X. Wang, "An Improved Activity Recognition Method Based on Smart Watch Data," 2017 IEEE International Conference on Computational Science and Engineering (CSE) and IEEE International Conference on Embedded and Ubiquitous Computing (EUC), Guangzhou, 2017, pp. 756-759.

[10] M. Babiker, O. O. Khalifa, K. K. Htike, A. Hassan and M. Zaharadeen, "Automated daily human activity recognition for video surveillance using neural network," 2017 IEEE 4th International Conference on Smart Instrumentation, Measurement and Application (ICSIMA), Putrajaya, 2017, pp. 1-5.

[11] H. F. Nweke, Y. W. Teh, M. A. Al-garadi and U. R. Alo, "Deep Learning algorithms for human activity recognition using mobile and wearable sensor networks: State of the art and research challenges," Expert Systems with Applications, vol. 105, pp. 233-261, 2018.

[12] X. Shi, Y. Li, F. Zhou and L. Liu, "Human Activity Recognition Based on Deep Learning Method," 2018 International Conference on Radar (RADAR), Brisbane, QLD, 2018, pp. 1-5.

[13] N. H. Friday, M. A. Al-garadi, G. Mujtaba, U. R. Alo and A. Waqas, "Deep learning fusion conceptual frameworks for complex human activity recognition using mobile and wearable sensors," 2018 International Conference on Computing, Mathematics and Engineering Technologies (iCoMET), Sukkur, 2018, pp. 1-7.

[14] Y. Chen and Y. Xue, "A Deep Learning Approach to Human Activity Recognition Based on Single Accelerometer," 2015 IEEE International Conference on Systems, Man, and Cybernetics, Kowloon, 2015, pp. 1488-1492.

[15] T. Perumal, Y. L. Chui, M. A. B. Ahmadon and S. Yamaguchi, "IoT based activity recognition among smart home residents," 2017 IEEE 6th Global Conference on Consumer Electronics (GCCE), Nagoya, 2017, pp. 1-2.

[16] L. R. Rabiner, "A tutorial on hidden markov models and selected applications in speech recognition", Proceedings of the IEEE, vol. 77, no. 2, pp. 257-286, Feb. 1989.

[17] S. Hochreiter and J. Schmidhuber, "Long Short-term Memory", Neural Computation, vol. 9, pp. 1735-1780, November 1997.

[18] Forkan, I. Khalil, Z. Tari, S. Foufou, and A. Bouras, “A Context-aware Approach for Long-term Behavioural Change Detection and Abnormality Prediction in Ambient Assisted Living," Pattern Recognition, vol. 48, no. 3, pp. 628-641, 2015.

[19] Y. F. Tan, S. C. Poh, X. Guo, S. N. Cheong, C. P. Ooi and W. H. Tan, "LSTM and HMM comparison for home activity anomaly detection," 2019 IEEE 3rd Information Technology, Networking, Electronic and Automation Control Conference, 15-17 March 2019, Chengdu, China. (accepted) 
[20] S. C. Poh, Y. F. Tan, S. N. Cheong, C. P. Ooi and W. H. Tan, "Abnormal detection for home activity based on sequence pattern," International Conference on Advanced Science, Engineering and Technology, 20-21 March 2019, Cyberjaya, Malaysia. (accepted)

[21] M. Ester, H. Kriegel, J. Sander and X. Xu, "A density-based algorithm for discovering clusters in large spatial databases with noise," Proceedings of the Second International Conference on Knowledge Discovery and Data Mining (KDD-96), pp. 226-231, 1995.

[22] E. Hoque, R. F. Dickerson, S. M. Preum, M. Hanson, A. Barth and J. A. Stankovic, "Holmes: A Comprehensive Anomaly Detection System for Daily In-home Activities," 2015 International Conference on Distributed Computing in Sensor Systems, Fortaleza, pp. 40-51, 2015.

[23] D. Arifoglu, and A. Bouchachia, "Activity Recognition and Abnormal Behaviour Detection with Recurrent Neural Networks,” Procedia Computer Science, vol. 110, pp. 86-93, 2017.

[24] D. Riboni, C. Bettini, G. Civitarese, Z. H. Janjua, and R. Helaoui, et al.,"SmartFABER: Recognizing Fine-grained Abnormal Behaviors for Early Detection of Mild Cognitive Impairment," Artificial Intelligence in Medicine, vol. 67, pp. 57-64, 2016.

[25] D. Cook, Aruba, Washington State University, Center for Advanced Studies in Adaptive Systems, 2011. Available at: http://casas.wsu.edu/datasets/.

\section{BIOGRAPHIES OF AUTHORS}

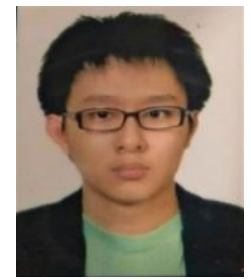

Soon-Chang Poh obtained his B. Eng. (Hons.) Electronics from Multimedia University, Malaysia. He is currently a research officer at Faculty of Engineering, Multimedia University.

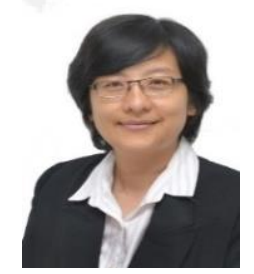

Dr. Yi-Fei Tan obtained her B.Sc. (Hons), M.Sc. and PhD from University of Malaya (UM). She is currently a senior lecturer at the Faculty of Engineering in Multimedia University (MMU), Cyberjaya, Malaysia. Her research interests include machine learning, image processing, big data analytics and queueing theory.

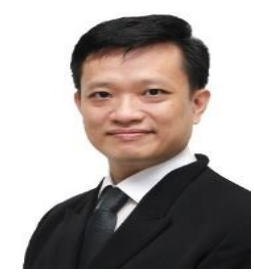

Soon-Nyean Cheong received his B.Eng. (Hons) and his Master of Engineering Science from Multimedia University, Malaysia. He is currently a senior lecturer at Faculty of Engineering, Multimedia University, Malaysia. His teaching and research interests include web engineering, natural user interface, smarthome, gerontechnology, educational technology and interactive mulimedia content.

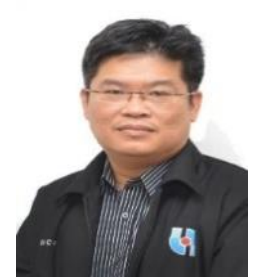

Dr. Chee-Pun Ooi obtained his Ph.D. in Electrical Engineering from University of Malaya, Malaysia, in year 2010. Dr. Ooi current position is senior lecturer with Faculty of Engineering in Multimedia University. He is a chartered engineer registered with Engineering Council (the British regulatory body for Engineers), member of IET. His research areas are in FPGA based embedded systems, embedded systems.

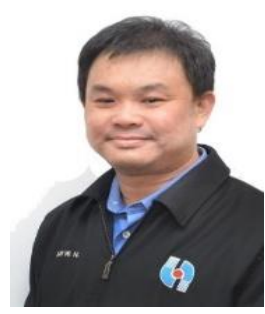

Dr. Wooi-Haw Tan received his M.Sc. in Electronics from Queen's University of Belfast, UK and a Ph.D. in Engineering from Multimedia University. He is currently a senior lecturer at Multimedia University. Dr. Tan's areas of expertise include image processing, embedded system design, Internet of Things (IoT), machine learning and deep learning. He is teaching embedded system design and computer networking at both undergraduate and postgraduate levels, as well as providing training, workshops and short courses for industries especially in the areas of Internet of Things (IoT), machine learning and deep learning. His research works have been published in numerous international journals and conferences. Besides, he has also co-authored two textbooks on microcontroller systems. 